\title{
SE JUSTIFICA EL SEGUIMIENTO DESPUÉS DE UNA COLECTOMÍA EN PACIENTES CON POLIPOSIS ADENOMATOSA FAMILIAR*
}

\author{
Drs. María Cristina García ${ }^{1}$, Eliana Pinto ${ }^{1}$, Udo Kronberg ${ }^{1}$, \\ Alejandro J. Zárate ${ }^{1}$, Francisco López-Köstner ${ }^{1}$ \\ 1 Unidad de Coloproctología Clínica Las Condes. \\ Santiago, Chile.
}

\begin{abstract}
The follow up after colectomy in patients with familial adenomatous polyposis is justified
\end{abstract}

Background: Patients with classic familial adenomatous polyposis syndrome (FAPc) have a germline mutation in the APC gene. The most common phenotypic expression is the presence of multiple colorectal adenomatous polyps (more than 100), with high probability developing colorrectal cancer (CRC) before the fifth decade of life. Prophylactic surgery (total colectomy or restorative proctocolectomy) reduces the risk of developing CRC. However, the risk of developing tumors in other organs remains present. Objetive: Analyze the frequency and type of tumors associated with classic familial adenomatous polyposis syndrome (FAPc) patients undergoing prophylactic colectomy. Material and Methods: Cohort study. From the registry of hereditary colorrectal cancer (CRC) at our institution, we identified patients with FAPc who underwent total colectomy with ileorrectal anastomosis (TC-IRA) or restorative proctocolectomy (RTPC), from 1999 to 2014. In the follow-up we analyzed related tumors and mortality. Results: 27 patients, of whom 18 (66.7\%) underwent TC-IRA and $9(33.3 \%)$ underwent RTPC. At the time of surgery, 4 patients had CRC $(15 \%)$ and 5 had extracolonic tumors (osteomas). In a mean follow-up of 49, 4 months (i: 2 y 178) the following lesions were diagnosed: digestive tract adenomas in $17(63 \%)$ patients, of these 2 required a proctectomy and 3 resection of duodenal adenomas. Eight patients developed desmoid tumors (30\%), and 3 of them underwent surgery. One patient had an extradigestive tumor (thyroid cancer) and only 8/27 (29.6\%) did not develop other tumors. One patient died due to progression of his CCR. Discussion: In this series it is confirmed that most patients will develop neoplasms FAPc after colectomy. Conclusion: The removal of the colon and/or rectum is able to prevent the development of CRC. However, two thirds of the patients develop other tumors in which systematic surveillance allowed early detection and treatment.

Key words: Familiar adenomatous polyposis, colorectal cáncer, desmoid tumor, duodenal cancer.

*Recibido el 10 de agosto de 2015 y aceptado para publicación el 5 de octubre de 2015.

Conflicto de intereses: ninguno

Correspondencia: Dr. Francisco López-Köstner.

flopez@clc.cl 


\section{Resumen}

Objetivo: Analizar la frecuencia y tipo de tumores asociados en pacientes con poliposis adenomatosa familiar clásica (PAFc) sometidos a una colectomía profiláctica. Materiales y Métodos: Estudio de cohorte. Desde el registro de cáncer colorrectal (CCR) hereditario, se identificaron las familias con PAFc, y de estas a los pacientes que se les practicó una colectomía total con anastomosis íleorrectal (CT-AIR) o proctocolectomía restauradora (PCTR), desde 1999 al 2014. En el seguimiento se analizaron los tumores asociados y su mortalidad. Resultados: Se identificaron 27 pacientes, de los cuales $18(66,7 \%)$ fueron sometidos a CT-AIR y $9(33,3 \%)$ a PCTR. Al momento de la cirugía, 4 pacientes presentaban CCR $(15 \%)$ y 5 tenían tumores extracolónicos (osteomas). En un seguimiento promedio de 49,4 meses (i: 2 y 178) se diagnosticaron: adenomas del tracto digestivo en $17(63 \%)$ pacientes, de éstos 2 requirieron una proctectomía y 3 resecciones de adenomas duodenales. Ocho pacientes desarrollaron tumores desmoides (30\%), y 3 de ellos fueron sometidos a una cirugía. Un paciente presentó un tumor extradigestivo (cáncer de tiroides) y sólo 8/27 (29,6\%) pacientes no desarrollaron otros tumores. Un paciente falleció por progresión de su CCR. Discusión: En esta serie se confirma que la mayoría de los pacientes con PAFc seguirán desarrollando neoplasias después de su colectomía. Conclusiones: La extirpación del colon y/o recto permitió evitar el desarrollo de CCR. Sin embargo, dos tercios de los pacientes presentaron otros tumores en quienes su seguimiento permitió una detección y tratamiento temprano.

Palabras clave: Poliposis adenomatosa familiar, cáncer colorrectal, tumores desmoides, adenomas duodenales.

\section{Introducción}

La poliposis adenomatosa familiar (PAF) es una patología autosómica dominante causada por mutaciones en el gen APC (cromosoma 5), y se caracteriza por la presencia de múltiples pólipos adenomatosos en el epitelio colorrectal y el desarrollo de otros tumores extracolónicos, donde el carcinoma de la papila de Vater es la segunda causa de muerte después del cáncer colorrectal ${ }^{1-4}$. Dentro de los tumores extra digestivos, los desmoides se presentan aproximadamente en un tercio de los pacientes sometidos a cirugía profiláctica, y se observan otros menos frecuentes como tiroides, osteomas, etc. ${ }^{1-4}$. Los pacientes con PAF clásica $(\mathrm{PAFc})$ desarrollan durante la adolescencia y adultez temprana cientos a miles de pólipos en el colon y recto, que -si se dejan sin tratamiento- casi invariablemente desarrollarán cáncer colorrectal (CCR) antes de la quinta década de la vida ${ }^{5}$. El conteo de pólipos mayor o igual a 1.000 confiere un riesgo relativo para CCR de 2,3 a los 40 años con una penetrancia cercana al $100 \%{ }^{6}$.

La cirugía profiláctica se considera el tratamiento de elección en PAFc y está indicada al momento del diagnóstico sintomático o alrededor de los 20 años de vida para reducir el riesgo de desarrollar CCR. Las opciones terapéuticas son principalmente dos: la colectomía total más íleo-recto anastomosis (IRA) y la proctocolectomía restauradora con reservorio ileal. En centros de referencia, la proctocolectomía con ileostomía terminal, es cada vez menos frecuente. La decisión de cuál técnica utilizar depende de varios factores como el compromiso rectal con adenomas, la posibilidad de seguimiento endoscópico periódico y el deseo de fertilidad, ya que la extirpación del recto se acompaña de una reducción de la fertilidad ${ }^{8,9}$.

Independiente de cuál sea el procedimiento quirúrgico elegido, estos pacientes requieren evaluaciones periódicas del recto remanente, por una incidencia de cáncer en el muñón rectal de $25 \%$ a los 70 años de edad, con una tasa de proctectomía posterior de hasta el $60 \%$ a los 70 años $^{9,10}$. Por otra parte, en el reservorio ileal la posibilidad de desarrollar adenomas se incrementa con la edad del reservorio, y a los 15 años se estima que es de hasta el $75 \%$, aunque el desarrollo de cáncer es infrecuente ${ }^{11,12}$. En la anastomosis reservorio-anal, existe la posibilidad de formación de adenomas con displasia y adenocarcinomas a largo plazo $^{13}$, cuando queda mucosa rectal remanente.

El desarrollo de registros genéticos y programas de alto riesgo ha demostrado incrementar la expectativa de vida de estos pacientes ${ }^{14}$. Esta mayor sobrevida ha promovido que se identifiquen otras patologías asociadas a las mutaciones, las cuales se convierten en la principal causa de morbilidad y mortalidad a largo plazo una vez resecado el colon y el recto.

Entre las manifestaciones extra intestinales están los tumores desmoides con incidencias reportadas alrededor de un $20 \%-30 \%{ }^{15}$, además de los osteomas y anomalías dentarias que tienen una incidencia similar. Aunque con una menor incidencia, pero igualmente importantes por su potencial de morbilidad y letalidad hay otras patologías como los tumores de 
tiroides (principalmente el cáncer papilar), tumores hepatobiliares (hepatoblastoma-colangiocarcinomatumores pancreáticos) y tumores del sistema nervioso central (meduloblastoma) entre otros.

El objetivo de este trabajo es analizar la frecuencia y el tipo de tumores asociados en una cohorte de pacientes sometidos a una colectomía profiláctica por PAFc.

\section{Material y Métodos}

Estudio de cohorte histórica, descriptivo. Desde el registro de CCR hereditario, se identificaron las familias con PAFc y de éstas los pacientes a quienes se les practicó una colectomía total o proctocolectomía restauradora, entre junio de 1999 y junio de 2014.

El protocolo de seguimiento incluyó: Evaluación médica, endoscopia digestiva alta inicial y de seguimiento de acuerdo a la clasificación de Spigelman ${ }^{15}$ la cual se muestra en la Tabla 1, colonoscopia inicial y posteriormente recto/reservorioscopia anual, tomografía computada de abdomen y pelvis periódica de acuerdo a síntomas o presencia de tumores desmoides y ecografía tiroidea periódica.

Los datos del seguimiento se extrajeron del registro y de las historias clínicas de cada paciente.

De las historias clínicas se registraron los datos demográficos de los pacientes, fecha y edad a la que se practicó la cirugía, el tiempo de seguimiento y el desarrollo de nuevos tumores:

1. CCR previo a la cirugía.

2. CCR posterior a la cirugía.

3. Tumores desmoides intra y extraabdominales.

4. Osteomas cráneo faciales.

5. Tumores de tiroides (benignos y malignos).

6. Tumores de sistema nervioso central.

7. Tumores de páncreas.

8. Tumores suprarrenales.

9. Desarrollo de nuevos adenomas del tracto digestivo (estómago, duodeno, remanente rectal, reservorio ileal).
10. Otros (se incluyeron otros diagnósticos que no estuviesen especificados en los desenlaces principales).

11. Mortalidad.

Los resultados se reportan en números absolutos y relativos porcentuales.

\section{Resultados}

Se identificaron 27 pacientes pertenecientes a 23 familias, de los cuales 16 fueron mujeres y 11 hombres. El promedio de edad fue de 30,1 años (i: 8-68 años).

A 18 pacientes $(67 \%)$ se les practicó una colectomía más IRA y a 9 pacientes $(33 \%)$ una proctocolectomía más reservorio ileal con anastomosis reservorio anal.

En los pacientes con colectomía y anastomosis íleorrectal, se observaron nuevos adenomas del recto remanente en 8/18 (45\%) requiriendo polipectomías endoscópicas. En dos pacientes fue necesario practicar una proctectomía por el desarrollo de poliposis severa, sin diagnóstico de CCR en ninguno de ellos.

De los 9 pacientes con proctocolectomía más reservorio ileal con anastomosis reservorio anal, uno $(11 \%)$ desarrolló adenomas en el reservorio que se extirparon endoscópicamente.

El tiempo promedio de seguimiento fue de 49,4 meses (i: 2 y 178). En la Tabla 2 se muestran los tumores que se identificaron en esta serie.

Se diagnosticaron 17 pacientes $(63 \%)$ con pólipos del tracto digestivo superior, con una mediana de aparición de 29 meses (rango entre 5 y 131) desde la cirugía. De estos, 11 pacientes presentaron adenomas duodenales, 3 pacientes ampulomas que requirieron ampulectomía endoscópica. En el análisis anatomopatológico de los ampulomas se evidenció adenomas tubulares con grados variables de displasia, ninguno de ellos con focos de carcinoma.

El segundo diagnóstico más frecuente en esta serie fueron los tumores desmoides con un total de

Tabla 1. Clasificación de Spigelman

\begin{tabular}{|llll|}
\hline & $\mathbf{1}$ punto & $\mathbf{2}$ puntos & $\mathbf{3}$ puntos \\
Número de pólipos & $1-4$ & $5-20$ & $>20$ \\
Tamaño & $1-4$ & $5-10$ & $>10$ \\
Histología & Tubular & Túbulo-velloso & Velloso \\
Displasia & Leve & Moderada & Severa \\
\hline
\end{tabular}

Estadio según puntuación: Estadio 0: 0 puntos. Estadio I: 1-4 puntos. Estadio II: 5-6 puntos. Estadio III: 7-8 puntos. Estadio IV: 9-12 puntos. 
Tabla 2. Diagnósticos asociados en el seguimiento

\begin{tabular}{|c|c|c|}
\hline TIPO DE TUMOR & $\mathbf{n}$ & $\%$ \\
\hline $\begin{array}{lr}\text { Adenomas TGI* } & \\
\text { Estómago: } & 5 \\
\text { Duodeno: } & 11 \\
\text { Recto/reservorio: } & 9\end{array}$ & 17 & 63 \\
\hline $\begin{array}{ll}\text { Tumores desmoides } \\
\text { Intraabdominales: } & 4 \\
\text { Extraabdominales: } & 1 \\
\text { Mixtos: } & 3\end{array}$ & 8 & 30 \\
\hline Tumores tiroideos & 1 & 4 \\
\hline Tumores suprarrenales & 2 & 7 \\
\hline $\begin{array}{l}\text { Otros tumores } \\
\text { Linfoma MALT: } \\
\text { Quiste epidermoide: } 1\end{array}$ & 2 & 7 \\
\hline
\end{tabular}

*TGI: Tracto gastrointestinal.

8 pacientes (30\%), con una mediana de aparición de 28 meses desde la cirugía (rango entre 12 y 97 meses). De acuerdo a su localización, 4 pacientes presentaron de raíz de mesenterio, un paciente un tumor de pared abdominal exclusivo y 3 pacientes tumores en ambas ubicaciones. En el seguimiento, $2 / 8(25 \%)$ pacientes han permanecido asintomáticos por lo que no han requerido tratamiento, dos pacientes reciben tratamiento con tamoxifeno con estabilización de la lesión. A un paciente con tumor retroperitoneal con compresión ureteral e hidronefrosis bilateral se le inició tratamiento farmacológico con regresión de la lesión y resolución de la obstrucción. En un paciente se practicó resección quirúrgica de un tumor intraabdominal sin evidencia de recidiva. Un paciente presentó múltiples lesiones en la raíz del mesenterio, una de ellas con compresión de los vasos mesentéricos con isquemia del reservorio ileal, por lo que requirió resección del mismo, además recibió tratamiento secuencial con tamoxifeno, vinorelbina y doxorubicina pegilada. En un segundo paciente se observaron tumores desmoides múltiples del mesenterio que desencadenaron una fístula entero-cutánea y de la pared abdominal, que han sido motivo de varias intervenciones quirúrgicas.

Tumores suprarrenales se identificaron en 2 pacientes $(7 \%)$ todos ellos en seguimiento, ninguno ha requerido tratamiento específico.

Un paciente desarrolló cáncer de tiroides en el seguimiento, con un patrón papilar que se resolvió con cirugía y actualmente se encuentra sin recaída en seguimiento oncológico.

Se identificaron 2 pacientes (7\%) con otros tumores entre los que se destaca un paciente con un linfo- ma MALT del intestino delgado como hallazgo en la patología quirúrgica, y un paciente con un quiste epidermoide. Cinco pacientes presentaron osteomas cráneo-faciales.

No se encontraron pacientes con tumores pancreáticos, de $\mathrm{SNC}$ o hepatobiliares.

En los 4 pacientes (15\%) que al momento de la cirugía tenían CCR, sólo uno de ellos quien tenía un CCR estadio III, presentó metástasis pulmonares a los 3 años de su cirugía y posteriormente falleció.

\section{Discusión}

En esta serie se confirma que la mayoría de los pacientes con PAFc seguirán desarrollando neoplasias después de su colectomía. En el muñón rectal, el riesgo de CCR es de $25 \%$ a los 70 años de edad, con una tasa de proctectomía posterior de hasta el $60 \%$ a los 70 años ${ }^{9,10}$. En esta serie se logró identificar pólipos del recto remanente en el $45 \%$ de los pacientes a quienes se les había practicado una colectomía más IRA, y la mayoría de ellos fue tratado endoscópicamente. Sólo se requirió la proctectomía en 2 pacientes. A uno de ellos se le había practicado la colectomía más IRA a los 20 años de edad, y en el seguimiento desarrolló una poliposis severa del recto remanente por lo que 11 años después de su primera cirugía se realiza la proctectomía. El segundo caso es un paciente a quien se le practica su cirugía profiláctica a los 8 años de edad por una poliposis severa del colon, con preservación de recto y 8 años más tarde se completa la proctectomía por compromiso severo del recto remanente. En ambos pacientes se reconstituyó el tránsito con un reservorio ileal y en ningún caso se observó el desarrollo de CCR.

En esta serie se practicó la proctocolectomía más reconstrucción con reservorio ileal en el 33\% de los pacientes. En el reservorio ileal también existe la posibilidad de desarrollar adenomas y se describen varios factores de riesgo de los cuales los más importantes parecen ser la presencia de más de 1.000 pólipos al momento de la cirugía y la edad del reservorio, a los 15 años se estima que es de hasta el $75 \%$, aunque el desarrollo de cáncer parece ser raro $^{11,12}$. En esta serie se detectó un paciente con adenomas en el reservorio ileal. Estos datos son menores a lo reportado en la literatura, sin embargo, es lo esperado ya que hasta el momento hay un seguimiento promedio de 49 meses, y el número de pacientes es limitado.

En esta serie se detectó una alta incidencia de pólipos de glándulas fúndicas $(10 / 27 ; 37 \%)$ con baja frecuencia de adenomas gástricos (solamente el 19\% de los pacientes), similar a lo reportado en la literatura. No hubo casos de cáncer gástrico en el período 
evaluado. En series occidentales no se reporta una mayor incidencia de cáncer gástrico cuando se compara con la población general, mientras que en series de Japón y Corea ${ }^{16}$, se reporta una mayor incidencia de cáncer gástrico en los pacientes con PAF. Por lo anterior el seguimiento del tracto digestivo está marcado por la enfermedad duodenal más que por el desarrollo de pólipos gástricos. No obstante, se recomienda la resección y biopsia de los adenomas gástricos de acuerdo a su apariencia endoscópica como parte del seguimiento ${ }^{4}$.

Se menciona en la literatura que los pacientes con PAF tienen un riesgo cercano al $100 \%$ de desarrollar adenomas duodenales a lo largo de la vida, en esta serie la incidencia fue de $41 \%$ que es comparable con lo reportado en otras series donde se mencionan incidencias que varían entre el $58 \%$ y el $74 \%{ }^{17}$. Los pacientes con PAF tienen un riesgo mayor que la población general de desarrollar cáncer duodenal siendo de aproximadamente un 5\% con una incidencia acumulada a los 57 años de 4,5\% ${ }^{18}$. En los pacientes con una clasificación de Spiegelman de IV, la incidencia es de aproximadamente $36 \%$ comparado con un $2 \%$ en los pacientes con Spiegelman II y III ${ }^{4,19}$. En esta serie no se diagnosticó ningún cáncer duodenal en el período evaluado. En tres de nuestros pacientes se practicó una ampulectomía endoscópica por adenomas de la papila duodenal, ninguno de ellos con adenocarcinoma en el análisis patológico.

Los tumores desmoides representaron el segundo tumor asociado más frecuente en esta serie. Este es un tumor de presentación infrecuente en la población general, pero se observa entre el $10 \%$ y $15 \%$ de los pacientes con PAF con mayor frecuencia después de procedimientos quirúrgicos con incidencias de entre el $30 \%$ y $75 \%{ }^{20}$. En esta serie, el desarrollo de estos tumores fue en todos los casos posterior a la cirugía inicial con una incidencia del 30\% similar a lo reportado en la literatura. Este tumor se considera benigno porque carece de potencial metastásico aunque su comportamiento en general es incierto ya que puede variar entre la estabilidad o incluso regresión en el seguimiento, y por otro lado hay tumores con comportamiento agresivo con invasión local de órganos y tejidos. En esta serie los tumores mostraron el espectro completo, es decir, un $25 \%$ presentó un curso agresivo de su tumor, $25 \%$ ha tenido tumores asintomáticos, $25 \%$ ha tenido lesiones que han permanecido estables con el tratamiento médico, $12,5 \%$ presentó regresión y otro $12,5 \%$ se resolvió con tratamiento quirúrgico.

Solamente se identificó un paciente con cáncer de tiroides (incidencia de 3,7\%), con un patrón papilar que es el tumor que se asocia con mayor frecuencia a la PAFc, y el paciente recibió tratamiento oportu- no, sin complicaciones. En la literatura se menciona un riesgo de aproximadamente $2 \%$ de padecer cáncer de tiroides a lo largo de la vida ${ }^{2,21}$, Herraiz M y cols. $^{21}$, encontraron una incidencia del $12 \%$ en su serie de 51 pacientes, nuestra incidencia fue menor probablemente en relación al número de pacientes en seguimiento.

En esta serie no se identificaron tumores hepatobiliares, de sistema nervioso central, ni de páncreas, que en general tienen bajas incidencias.

Es importante destacar el papel del registro familiar y asesoramiento genético de acuerdo al riesgo definido en los portadores. La consejería genética involucra recopilar, documentar y evaluar el riesgo basado en la historia familiar y personal, tanto del caso índice como de los otros miembros de la familia. Un apropiado seguimiento de los individuos con riesgo aumentado de cáncer reduce la incidencia de los cánceres asociados con la condición heredada. Numerosos estudios han demostrado que es costo-efectivo con una reducción en la mortalidad global $^{22-30}$. En los pacientes pertenecientes al registro de CCR hereditario esta evaluación de riesgo y seguimiento, permitió una búsqueda activa de nuevas neoplasias con el resultado que hasta el momento la detección se ha realizado en forma precoz por lo que el tratamiento oportuno se ha acompañado de una buena expectativa de vida.

\section{Referencias}

1. Guilherme F, Oliva R, Rocco A, Edmond V, Carlos $\mathrm{S}$, Cecconello I. Evaluating Causes of Death in Familial Adenomatous Polyposis. J Gastrointest Surg. 2010;14:1943-9.

2. Groen EJ, Muntinghe FL, Enting RH, De Vries J, kleibeuker JH, Witjes MJ, et al. Extra-intestinal manifestations of Familial Adenomatous Polyposis. Annals of Surgical Oncology 2008;15:2439-50.

3. Kasper B, Hohenberger P. Desmoid Tumors: clinical features and treatment options for advanced disease. The Oncologist 2011;16:682-93.

4. Will OCC, Man RF, Phillips RKS, Tomlinson IP, Clarck SK. Familial adenomatous polyposis and the small bowel: A loco-regional review and current management strategies. Pathol Res Pract. 2008;204:449-58.

5. Macrae F. Familial Adenomatous Polyposis. Best Practice \& Research Clinical Gastroenterology 2009;23:197207.

6. Varesco L. Familial adenomatous poliposys: genetics and epidemiology. Tech Coloproctol. 2004;8:s305-s8.

7. Clark. Colorectal cancer: no longer de issue in familial adenomatous polyposis? Familial Cancer 2011;10:11-20.

8. Wu JS, Paul P, McGannon EA, Church JM. APC Genotype, Polyp Number, and Surgical Options in Familial Adenomatous Polyposis. Annals of Surgery 
1998;227:57-62.

9. McGrath DR, Spiegelman AD. In the Beginning There Was Colectomy: Current Surgical Options in Familial Adenomatous Polyposis. Hereditary Cancer in Clinical Practice 2004;2:153-60.

10. Campos FG, Pérez RO, Imperiale AR, Seid VE, Nahas SC, Cecconello I. Evaluating Causes of Death in Familial Adenomatous Polyposis. Journal of Gastrointestinal Surgery 2010;14:1943-9.

11. Tonelli F, Ficari F, Bargellini T, Valanzano R. Ileal Pouch Adenomas and Carcinomas After Restorative Proctocolectomy for Familial Adenomatous Polyposis. Diseases of the Colon and Rectum 2012;55:322-9.

12. Pommaret E, Vienne A, Lefevre JH, Sogni P, Florent C, Desaint B, et al. Prevalence and risk factors for adenomas in the ileal pouch and the afferent loop after restorative proctocolectomy for patients with familial adenomatous polyposis. Surgical Endoscopy 2013;27:3816-22.

13. Kartheuser A, Stangherlin P, Brandt D, Remue C, Sempoux C. Restorative proctocolectomy and ileal pouchanal anastomosis for familial adenomatous polyposis revisited. Familial Cancer 2006;5:241-60.

14. Wilding A, Fiona L, Clancy T, Huson S, Moran A, Evans DG. Life expentancy in hereditary cancer predisposing diseases: an observational study. J Med Genet 2012;49:264-9.

15. Spigelman AD, Talbot IC, Williams CB, Domizio P, Phillips RKS. Upper Gastrointestinal Cancer In Patients With Familial Adenomatous Polyposis. The Lancet 1989;2:783-5.

16. Park SY, Ryu JK, Park JH, Yoon H, Kim JY, Yoon YB, et al. Prevalence of Gastric and Duodenal Polyps and Risk Factors for Duodenal Neoplasm in Korean Patients with Familial Adenomatous Polyposis. Gut and Liver 2011;5:46-51.

17. Bülow S, Björk S, Christensen IJ, Fausa O, Järvinen H, Moesgaard F, et al. The DAF study Group. Duodenal adenomatosis in familial adenomatous poliposis. Gut 2004;53:381-6.

18. Gallagher MC, Phillips RKS, Bulow S. Surveillance and management of upper gastrointestinal disease in Familial Adenomatous Polyposis. Familial Cancer 2006;5:263-73.

19. Balmforth DC, Phillips RKS. Advanced disease in familial adenomatous polyposis: How frecuently should patients be followed up after successful therapy? Familial Cancer 2012;11:553-7.

20. Church JM, McGannon E, Ozuner G. The clinical course of intra-abdominal desmoid tumours in patients with familial adenomatous polyposis. Colorectal Disease 1999;1:168-73.

21. Herraiz M, Barbesino G, Faquin W, Chan-Smutko G, Patel D, Shannon KM, et al. Prevalence of Thyroid Cancer in Familial Adenomatous Polyposis Syndrome and the Role of Screening Ultrasound Examinations. Clinical Gastroenterology and Hepatology 2007;5:36773

22. Bertario L, Sala P, Giarola M, Mondini P, Pierotti M, Spinelli $\mathrm{P}$, et al. Multiples approach to the exploration of genotype-phenotype correlations in Familial Adenomatous Polyposis. Journal of Clinical Oncology 2003;21:1698-707.

23. Jasperson KW. Genetic Testing by Cancer Site, Colon (Polyposis Syndromes). The Cancer Journal 2012;18:328-33.

24. Järvinen HJ, Aarnio M, Mustonen H, Aktan-Collan K, Aaltonen LA, Peltoma"ki P, et al. Controlled 15-Year Trial on Screening for Colorectal Cancer in Families With Hereditary Nonpolyposis Colorectal Cancer. Gastroenterology 2000;118:829-34.

25. De Jong AE, Hendriks YMC, Kleibeuker JH, De Boer SY, Cats A, Griffioen G, et al. Decrease in Mortality in Lynch Syndrome Families Because of Surveillance. Gastroenterology 2006;130:665-71.

26. Järvinen HJ, Mecklin JP, Sistonen P. Screening Reduces Colorectal Cancer Rate in Families With Hereditary Nonpolyposis Colorectal Cancer. Gastroenterology 1995; 108:1405-11.

27. Solomon $\mathrm{CH}$, Pho LN, Burt RW. Current Status of Genetic Testing for Colorectal Cancer Susceptibility. Oncology 2002;16:161-71.

28. Soto G, López-Köstner F, Zárate AJ, Vuletin F, Rahmer A, León F, Zúiga A. Poliposis Adenomatosa Familiar. Alternativas Terapéuticas y Estudio de los Familiares. Rev Med Chile 2005;103:1043-150.

29. Soto G, López-Köstner F, Zárate AJ, Vúletin F, Zúñiga A, Rahmer A. Resultados Alejados en Poliposis Adenomatosa Familiar. Rev Chil Cir. 2003;55:149-54.

30. Azolas C, Bocic G, Jensen C, Garrido R, Pérez G. Poliposis Familiar: Estudio Familiar. Rev Chil Cir. 1994;46:418-22. 